\title{
Surjective isometries on spaces of differentiable vector-valued functions
}

\author{
by \\ Fernanda Botelho and James Jamison (Memphis, TN)
}

\begin{abstract}
This paper gives a characterization of surjective isometries on spaces of continuously differentiable functions with values in a finite-dimensional real Hilbert space.
\end{abstract}

1. Introduction. We consider the space of continuously differentiable functions on the interval $[0,1]$ with values in a Banach space $E$. This function space, equipped with the norm $\|f\|_{1}=\max _{x \in[0,1]}\left\{\|f(x)\|_{E}+\left\|f^{\prime}(x)\right\|_{E}\right\}$, is a Banach space, denoted by $C^{(1)}([0,1], E)$.

Banach and Stone obtained the first characterization of the isometries between spaces of scalar-valued continuous functions (see $[2,15])$. Several researchers derived extensions of the Banach-Stone theorem to a variety of different settings. For a survey of this topic we refer the reader to [7]. Cambern and Pathak $[4,5]$ considered isometries on spaces of scalarvalued differentiable functions and gave a representation for the surjective isometries of such spaces. In this paper, we extend their result to the vector-valued function space $C^{(1)}([0,1], E)$, for $E$ a finite-dimensional Hilbert space. We also characterize the generalized bi-circular projections on $C^{(1)}([0,1], E)$.

The characterization of the extreme points of the dual unit ball of a closed subspace of the continuous functions a compact Hausdorff space due to Arens and Kelley [6, p. 441] plays a crucial role in our proofs. In addition, the following result by de Leeuw which gives a converse of the Arens-Kelley theorem, for a closed subspace $\mathcal{X}$ of $C(\Omega)$ (cf. [11]), is also essential to our methods. To state de Leeuw's result we need the following definition.

Definition 1.1. The point $\omega \in \Omega$ is said to be a peak point for $h \in \mathcal{X}$ if $h(\omega)=1,\left|h\left(\omega_{1}\right)\right| \leq 1$ for every $\omega_{1} \in \Omega$, and $\left|h\left(\omega_{1}\right)\right|=1$ at some $\omega_{1} \neq \omega$ if and only if $\left|g\left(\omega_{1}\right)\right|=|g(\omega)|$ for all $g \in \mathcal{X}$. 
Theorem 1.2 (cf. [11, p. 61]). If $\omega \in \Omega$ is a peak point for some $h \in \mathcal{X}$, then the functional $\Phi \in \mathcal{X}^{*}$ defined by $\Phi(g)=g(\omega)$ is an extreme point of the unit ball in $\mathcal{X}^{*}$.

We construct an isometric embedding of $C^{(1)}([0,1], E)$ onto a closed subspace of the space of scalar-valued continuous functions on a compact set. This allows us to describe the form of the extreme points of $C^{(1)}([0,1], E)_{1}^{*}$. We denote by $B_{1}$ the unit ball in a Banach space $B$. We consider the isometry $F$ from $C^{(1)}([0,1], E)$ onto a subspace $\mathcal{M}$ of the scalar-valued continuous functions on $\Omega=[0,1] \times E_{1}^{*} \times E_{1}^{*}$, with $E^{*}$ equipped with the weak* topology,

$$
F(f)=F_{f}(x, \varphi, \psi)=\varphi(f(x))+\psi\left(f^{\prime}(x)\right) .
$$

The surjective isometry on the dual spaces $F^{*}\left(F_{f}^{*}\right)(g)=F_{f}^{*}\left(F_{g}\right)$ maps the extreme points of $\mathcal{M}_{1}^{*}$ onto the extreme points of $C^{(1)}([0,1], E)_{1}^{*}$. It follows from the Arens-Kelley lemma [6, p. 441] that

$$
\operatorname{ext}\left(\mathcal{M}_{1}^{*}\right) \subseteq\left\{\Phi_{\omega}: \Phi_{\omega}\left(F_{f}\right)=\varphi(f(x))+\psi\left(f^{\prime}(x)\right), \forall f \in C^{(1)}([0,1], E)\right\} .
$$

Proposition 1.3. If $E$ is a smooth, separable and reflexive Banach space, over the reals or complex numbers. Then $\Phi$ is an extreme point of $\mathcal{M}_{1}^{*}$ if and only if there exists $(x, \varphi, \psi) \in \Omega$, with $\varphi$ and $\psi$ extreme points of $E_{1}^{*}$, such that

$$
\Phi(f)=\varphi(f(x))+\psi\left(f^{\prime}(x)\right) .
$$

Proof. If $\Phi$ is an extreme point of $\mathcal{M}_{1}^{*}$, then $\Phi=\Phi_{\omega}$ for some $\omega=$ $(x, \varphi, \psi) \in \Omega$. If $\varphi$ (or $\psi)$ is not an extreme point of $E_{1}^{*}$, then there must exist distinct functionals $\varphi_{1}$ and $\varphi_{2}$ in $E_{1}^{*}$ such that $\varphi=\left(\varphi_{1}+\varphi_{2}\right) / 2$. For $i=1,2$, we set $\omega_{i}=\left(x, \varphi_{i}, \psi\right)$ and

$$
\Phi_{\omega_{i}}\left(F_{f}\right)=\varphi_{i}(f(x))+\psi\left(f^{\prime}(x)\right) .
$$

We have $\Phi=\left(\Phi_{\omega_{1}}+\Phi_{\omega_{2}}\right) / 2$ and

$$
\left|\Phi_{\omega_{i}}\left(F_{f}\right)\right| \leq\left|\varphi_{i}(f(x))\right|+\left|\psi\left(f^{\prime}(x)\right)\right| \leq\|f(x)\|_{E}+\left\|f^{\prime}(x)\right\|_{E} \leq\|f\|_{1}=\left\|F_{f}\right\|_{\infty} .
$$

On the other hand, there exist $\mathbf{a}_{i} \in E_{1}(i=1,2)$ so that $\left|\varphi_{i}\left(\mathbf{a}_{i}\right)\right|=1$. Thus, if $f_{i}$ is the constant function equal to $\mathbf{a}_{i}$, then $\left|\Phi_{\omega_{i}}\left(F_{f_{i}}\right)\right|=1$ and $\Phi_{\omega_{i}} \in \mathcal{M}_{1}^{*}$. Thus $\Phi$ is not an extreme point of $\mathcal{M}_{1}^{*}$, contradicting our initial assumption. Similar reasoning applies if $\psi \notin \operatorname{ext}\left(E_{1}^{*}\right)$.

Now we show that $\Phi$ given by

$$
\Phi(f)=\varphi(f(x))+\psi(f(x)),
$$

with $\omega=(x, \varphi, \psi) \in \Omega$ and $\varphi, \psi \in \operatorname{ext}\left(E_{1}^{*}\right)$, is an extreme point of $\mathcal{M}_{1}^{*}$. There exist $\mathbf{a}_{1}$ and $\mathbf{a}_{2}$ in $E_{1}$ such that $\varphi\left(\mathbf{a}_{1}\right)=e^{i \alpha_{1}}$ and $\psi\left(\mathbf{a}_{2}\right)=e^{i \alpha_{2}}$. We define $f \in C^{(1)}([0,1], E)$ by

$$
f(t)=\frac{e^{-i \alpha_{1}} \mathbf{a}_{1}+\lambda(t) e^{-i \alpha_{2}} \mathbf{a}_{2}}{2}
$$


with

$$
\lambda(t)= \begin{cases}-\frac{1}{2}\left(x^{2}-t^{2}\right)+(x-1)(x-t) & \text { for } 0 \leq t \leq x \\ -\frac{1}{2}\left(t^{2}-x^{2}\right)+(x+1)(t-x) & \text { for } x \leq t \leq 1\end{cases}
$$

We observe that $\lambda(x)=0, \lambda^{\prime}(x)=1$, and $|\lambda(t)|+\left|\lambda^{\prime}(t)\right|=1-\frac{1}{2}(x-t)^{2}<1$ for all $t \neq x$. Therefore

$$
\left|F_{f}(\omega)\right|=\left|\varphi(f(x))+\psi\left(f^{\prime}(x)\right)\right|=1 .
$$

If $\omega_{1} \neq \omega$ with $\omega_{1}=\left(x_{1}, \varphi_{1}, \psi_{1}\right)$ and $x_{1} \neq x$, we have

$$
\begin{aligned}
\left|F_{f}\left(\omega_{1}\right)\right| & =\left|\varphi_{1}\left(f\left(x_{1}\right)\right)+\psi_{1}\left(f^{\prime}\left(x_{1}\right)\right)\right| \\
& =\left|\varphi_{1}\left(\frac{e^{-i \alpha_{1}} \mathbf{a}_{1}+\lambda\left(x_{1}\right) e^{-i \alpha_{2}} \mathbf{a}_{2}}{2}\right)+\psi_{1}\left(\frac{\lambda^{\prime}\left(x_{1}\right) e^{-i \alpha_{2}} \mathbf{a}_{2}}{2}\right)\right| \\
& \leq \frac{1}{2}+\frac{\left|\lambda\left(x_{1}\right)\right|+\left|\lambda^{\prime}\left(x_{1}\right)\right|}{2}<1 .
\end{aligned}
$$

If $x_{1}=x$, and $\varphi_{1} \neq \varphi$ or $\psi_{1} \neq \psi$, then

$$
\begin{aligned}
\left|F_{f}\left(\omega_{1}\right)\right| & =\left|\varphi_{1}\left(f\left(x_{1}\right)\right)+\psi_{1}\left(f^{\prime}\left(x_{1}\right)\right)\right| \\
& =\left|\varphi_{1}\left(\frac{e^{-i \alpha_{1}} \mathbf{a}_{1}}{2}\right)+\psi_{1}\left(\frac{e^{-i \alpha_{2}} \mathbf{a}_{2}}{2}\right)\right|<1,
\end{aligned}
$$

unless $\left|\varphi_{1}\left(e^{-i \alpha_{1}} \mathbf{a}_{1}\right)\right|=1$ and $\left|\psi_{1}\left(e^{-i \alpha_{2}} \mathbf{a}_{2}\right)\right|=1$. The conclusion now follows from Theorem 1.2.

An extreme point of $\mathcal{M}_{1}^{*}$ is therefore represented by a triplet $(x, \varphi, \psi) \in$ $\Omega$, with $x \in[0,1]$ and $\varphi, \psi$ extreme points of $E_{1}^{*}$. Given the hypothesis on $E$ we know that $\operatorname{ext}\left(E_{1}^{*}\right)=E_{1}^{*}$. If $T$ is a surjective isometry of $C^{(1)}([0,1], E)$, then $T^{*}$ maps extreme points to extreme points. Hence Proposition 1.3 asserts that given $\omega=(x, \varphi, \psi)$ there exists $\omega_{1}=\left(x_{1}, \varphi_{1}, \psi_{1}\right)$ such that

$$
\varphi[(T f)(x)]+\psi\left[(T f)^{\prime}(x)\right]=\varphi_{1}\left(f\left(x_{1}\right)\right)+\psi_{1}\left(f^{\prime}\left(x_{1}\right)\right)
$$

for every $f \in C^{(1)}(\Omega, E)$.

This determines a transformation $\tau$, on $\Omega=[0,1] \times E_{1}^{*} \times E_{1}^{*}$, associated with the isometry $T$ and given by

$$
\tau(x, \varphi, \psi)=\left(x_{1}, \varphi_{1}, \psi_{1}\right) .
$$

LEMMA 1.4. $\tau$ is a homeomorphism.

Proof. We first observe that $\tau$ is well defined. Suppose there exist two triplets $\omega_{1}=\left(x_{1}, \varphi_{1}, \psi_{1}\right)$ and $\omega_{2}=\left(x_{2}, \varphi_{2}, \psi_{2}\right)$, both corresponding to $\omega=$ $(x, \varphi, \psi)$. Then

$$
\varphi_{1}\left(f\left(x_{1}\right)\right)+\psi_{1}\left(f^{\prime}\left(x_{1}\right)\right)=\varphi_{2}\left(f\left(x_{2}\right)\right)+\psi_{2}\left(f^{\prime}\left(x_{2}\right)\right) .
$$

If $x_{1} \neq x_{2}$, we select a function $f \in C^{(1)}([0,1], E)$ constant equal to a, an arbitrary vector in $E_{1}$, on a neighborhood of $x_{1}$, say $\mathcal{O}_{x_{1}}$, and equal to 
zero on a neighborhood of $x_{2}$, say $\mathcal{O}_{x_{2}}$, with $\mathcal{O}_{x_{1}} \cap \mathcal{O}_{x_{2}}=\emptyset$. Equation (1.3) implies that $\varphi_{1}(\mathbf{a})=0$, so $\varphi=0$. This contradicts $\varphi \in E_{1}^{*}$ and shows that $x_{1}=x_{2}$. If $f$ is now chosen to be constant equal to $\mathbf{a}$, an arbitrary vector in $E_{1}$, then (1.3) reduces to $\varphi_{1}(\mathbf{a})=\varphi_{2}(\mathbf{a})$, thus $\varphi_{1}=\varphi_{2}$. If $f$ is given by $f(x)=\left(x-x_{1}\right) \mathbf{a}$ then (1.3) implies that $\psi_{1}=\psi_{2}$. Therefore $\tau$ is well defined. Similar arguments and the invertibility of $T$ imply that $\tau$ is a bijection. The continuity of $\tau$ follows from the weak* continuity of $T^{*}$.

2. Properties of the homeomorphism $\tau$. In this section we explore further properties of the homeomorphism $\tau$ to be used in our characterization of surjective isometries on $C^{(1)}([0,1], E)$, with $E$ a real and finitedimensional Hilbert space.

For a fixed $x \in[0,1]$ we define the $\operatorname{map} \tau_{x}: E_{1}^{*} \times E_{1}^{*} \rightarrow[0,1]$ by $\tau_{x}(\varphi, \psi)=$ $\pi_{1} \tau(x, \varphi, \psi)$, with $\pi_{1}$ representing the projection on the first component.

The next lemma holds for a finite-dimensional Banach range space, the proof does not require an inner product structure.

Lemma 2.1. If E a finite-dimensional Banach space, then $\tau_{x}$ is a constant function.

Proof. If $\tau_{x}$ is not constant, then its image is a non-degenerate subinterval of $[0,1]$. We select a basis for $E^{*}$, say $\left\{\varphi_{1}, \ldots, \varphi_{k}\right\}$, consisting of functionals of norm 1 . We select an element

$$
y \in \tau_{x}\left(E_{1}^{*} \times E_{1}^{*}\right) \backslash\left\{\tau_{x}\left(\varphi_{i}, \varphi_{i}\right), \tau_{x}\left(\varphi_{i},-\varphi_{i}\right)\right\}_{i=1, \ldots, k} .
$$

Then we set $\tau\left(x, \varphi_{i}, \varphi_{i}\right)=\left(x_{i}, \eta_{i}, \xi_{i}\right), \tau\left(x, \varphi_{i},-\varphi_{i}\right)=\left(y_{i}, \alpha_{i}, \beta_{i}\right)$, and $\tau\left(x, \varphi_{0}, \psi_{0}\right)=(y, \eta, \xi)$. We select $g \in C^{(1)}([0,1], E)$ such that, for all $i=$ $1, \ldots, k, g\left(x_{i}\right)=g\left(y_{i}\right)=g^{\prime}\left(x_{i}\right)=g^{\prime}\left(y_{i}\right)=0_{E}, g(y)=u$ and $g^{\prime}(y)=v$, where $u$ and $v$ are such that $\eta(u)=1$ and $\xi(v)=1$. Therefore we have

$$
\varphi_{i}((T g)(x))+\varphi_{i}\left((T g)^{\prime}(x)\right)=\eta_{i}\left(g\left(x_{i}\right)\right)+\xi_{i}\left(g^{\prime}\left(x_{i}\right)\right)=0
$$

and

$$
\varphi_{i}((T g)(x))-\varphi_{i}\left((T g)^{\prime}(x)\right)=\alpha_{i}\left(g\left(y_{i}\right)\right)+\beta_{i}\left(g^{\prime}\left(y_{i}\right)\right)=0 .
$$

These equations imply that $\varphi_{i}((T g)(x))=0$ and $\varphi_{i}\left((T g)^{\prime}(x)\right)=0$ for all $i$. Hence $T g(x)=(T g)^{\prime}(x)=0_{E}$, implying that $2=\eta(g(y))+\xi\left(g^{\prime}(y)\right)=0$. This contradiction establishes the claim.

For fixed $x \in[0,1]$ and $\varphi \in E_{1}^{*}$, we define the map $\tau_{(x, \varphi)}: E_{1}^{*} \rightarrow E_{1}^{*}$ by

$$
\tau_{(x, \varphi)}(\psi)=\varphi_{1} \quad \text { provided that } \tau(x, \varphi, \psi)=\left(x_{1}, \varphi_{1}, \psi_{1}\right) .
$$

LEMMA 2.2. If $E$ is a finite-dimensional real Hilbert space then, for any fixed $x \in[0,1]$ and $\varphi \in E_{1}^{*}, \tau_{(x, \varphi)}$ is constant.

Proof. The Riesz Representation Theorem allows us to set notation as follows: $\varphi, \psi \in E_{1}^{*}$ are completely determined by the inner product with 
a single vector $u, v$ respectively. Hence we define $\tau:[0,1] \times E_{1} \times E_{1} \rightarrow$ $[0,1] \times E_{1} \times E_{1}$ by $\tau(x, u, v)=\left(x_{1}, u_{1}, v_{1}\right)$, and for every $f \in C^{(1)}([0,1], E)$,

$$
\langle(T f)(x), u\rangle+\left\langle(T f)^{\prime}(x), v\right\rangle=\left\langle f\left(x_{1}\right), u_{1}\right\rangle+\left\langle f^{\prime}\left(x_{1}\right), v_{1}\right\rangle .
$$

For fixed $x$ and $u$, we let $F_{(x, u)}: E_{1} \rightarrow E_{1}$ be given by $F_{(x, u)}(v)=$ $\pi_{2}(\tau(x, u, v))$, where $\pi_{2}$ is the projection on the second component. We prove the lemma by showing that $F_{(x, u)}$ is constant. For simplicity we denote $F_{(x, u)}$ by just $F$, unless the dependence on $x, u$ has to be emphasized.

We choose $f(t)=\mathbf{a}$, a unit vector. Then

$$
\langle(T f)(x), u\rangle+\left\langle(T f)^{\prime}(x), \pm v\right\rangle=\langle\mathbf{a}, F( \pm v)\rangle .
$$

This implies that

$$
\langle(T f)(x), u\rangle=\left\langle\mathbf{a}, \frac{F(v)+F(-v)}{2}\right\rangle
$$

for every $v \in E_{1}$. The function $G: E_{1} \rightarrow E$ defined by $G(v)=F(v)+F(-v)$ is therefore constant, denoted by $w$. As a consequence, for every $v_{0}$ and $v_{1}$ in $E_{1}$, we have

$$
\left\langle F\left(v_{0}\right), F\left(-v_{0}\right)\right\rangle=\left\langle F\left(v_{1}\right), F\left(-v_{1}\right)\right\rangle, \quad\left\langle F\left(v_{0}\right), F\left(v_{1}\right)\right\rangle=\left\langle F\left(-v_{0}\right), F\left(-v_{1}\right)\right\rangle .
$$

Therefore

$$
\left\|F\left(v_{0}\right)-F\left(-v_{0}\right)\right\|^{2}=2-2\left\langle F\left(v_{0}\right), F\left(-v_{0}\right)\right\rangle=2-2\left\langle F\left(v_{1}\right), F\left(-v_{1}\right)\right\rangle
$$

and

$$
\left\|F\left(v_{0}\right)-F\left(-v_{0}\right)\right\|^{2}=\left\|F\left(v_{1}\right)-F\left(-v_{1}\right)\right\|^{2} .
$$

Moreover, the function $H: E_{1} \rightarrow \mathbb{R}$ given by $H(v)=\|F(v)-F(-v)\|$ is also constant. This implies that

$$
\left\langle F\left(v_{0}\right)-F\left(-v_{0}\right), F\left(v_{0}\right)+F\left(-v_{0}\right)\right\rangle=0 .
$$

If $v \in E_{1}$ is such that $\{F(v), F(-v)\}$ is linearly independent, we set $\Pi_{v}$ to be the two-dimensional space spanned by $F(v)$ and $F(-v)$. Clearly $w \in \Pi_{v}$. In the plane $\Pi_{v}$, we represent $F(v)$ by $(w /\|w\|) e^{i \alpha}$ and $F(-v)$ by $(w /\|w\|) e^{-i \alpha}$. This is the polar representation for $F(v)$ and $F(-v)$ in $\Pi_{v}$, with $w$ identified with the positive direction of the $x$-axis. Without loss of generality, we choose $\alpha \in(0, \pi)$. This, in particular, implies that $w=F(v)+F(-v)=$ $(2 \cos (\alpha) /\|w\|) w$ and $2 \cos (\alpha)=\|w\|$. The value of $\alpha$ is then uniquely determined, so $\{F(v), F(-v)\}$ are the only two values in the range of $F$ belonging to the plane $\Pi_{v}$. The line $\overline{O w}$ divides the line segment $\overline{F(v) F(-v)}$ into two equal parts. Since $G$ is a constant function we have

$$
F\left(E_{1}\right) \subseteq\left(\frac{w}{2}+\{w\}^{\perp}\right) \cap \mathcal{S}\left(\frac{w}{2}, \frac{\|F(v)-F(-v)\|}{2}\right)
$$

with $\mathcal{S}(x, \delta)$ representing the set of points in $E$ at distance $\delta$ from $x \in E$, 
and $\{w\}^{\perp}$ representing the space orthogonal to the span of $w$. We also notice that $F\left(v_{0}\right) \neq \pm F\left(-v_{0}\right)$ for every $v_{0}$.

These considerations imply that $F$ maps the $n-1$-sphere ext $\left(E_{1}\right)$ to a set homeomorphic to a subset of an $n-2$-sphere, and $F$ sends antipodal points to antipodal points. We now show that such a map cannot exist.

First, for $n=2$ this would mean that $F$ would map $S^{1}$ onto two points, which is impossible since $S^{1}$ is connected and $F$ is continuous. The general case is a consequence of the Borsuk-Ulam Theorem (see [13, p. 266]).

Therefore $\{F(v), F(-v)\}$ is linearly dependent, and as a consequence, we consider the following two possibilities:

(i) $F(v)=F(-v)$ for every $v$,

(ii) $F(-v)=-F(v)$ for every $v$.

In case (i), we have $F(v)=w / 2$ for every $v$, so $F$ is constant.

In case (ii), given two different vectors $v_{0}$ and $v_{1}$ in $E_{1}$ we have

$$
\begin{aligned}
& \langle(T f)(x), u\rangle+\left\langle(T f)^{\prime}(x), \frac{v_{0}+v_{1}}{\left\|v_{0}+v_{1}\right\|}\right\rangle=\left\langle a, F\left(\frac{v_{0}+v_{1}}{\left\|v_{0}+v_{1}\right\|}\right)\right\rangle, \\
& \langle(T f)(x), u\rangle-\left\langle(T f)^{\prime}(x), \frac{v_{0}+v_{1}}{\left\|v_{0}+v_{1}\right\|}\right\rangle=\left\langle a, F\left(-\frac{v_{0}+v_{1}}{\left\|v_{0}+v_{1}\right\|}\right)\right\rangle .
\end{aligned}
$$

Hence $\langle(T f)(x), u\rangle=0$ and

$$
\left\langle(T f)^{\prime}(x), \frac{v_{0}+v_{1}}{\left\|v_{0}+v_{1}\right\|}\right\rangle=\left\langle a, F\left(\frac{v_{0}+v_{1}}{\left\|v_{0}+v_{1}\right\|}\right)\right\rangle .
$$

This implies that

$$
\frac{F\left(v_{0}\right)+F\left(v_{1}\right)}{\left\|v_{0}+v_{1}\right\|}=F\left(\frac{v_{0}+v_{1}}{\left\|v_{0}+v_{1}\right\|}\right), \quad\left\|F\left(v_{0}\right)+F\left(v_{1}\right)\right\|=\left\|v_{0}+v_{1}\right\| .
$$

Equation (2.2) implies that

$$
\left\langle F\left(v_{0}\right), F\left(v_{1}\right)\right\rangle=\left\langle v_{0}, v_{1}\right\rangle,
$$

or $F$ is norm preserving. We define a map $\Theta:[0,1] \times E_{1} \rightarrow C\left(E_{1}, E_{1}\right)$ by $\Theta(x, u)(v)=F_{(x, u)}(v)$. It follows from Lemma 1.4 that $\Theta$ is continuous. Furthermore, we have shown that, for each $(x, u) \in[0,1] \times E_{1}, \Theta(x, u)$ is either constant or an isometry in $E_{1}$.

The continuity of $\Theta$ and the connectedness of the domain $[0,1] \times E_{1}$ implies that the range of $\Theta$ consists only of constant functions on $C\left(E_{1}, E_{1}\right)$ or only of norm preserving functions on $E_{1}$ that map antipodal points onto antipodal points. This last assertion follows from the fact that the distance between one such norm preserving map on $E_{1}$ and a constant function is greater than or equal to $\sqrt{2}$. In fact, let $F_{x_{0}, u_{0}}=\Theta\left(x_{0}, u_{0}\right)$ be a constant function, everywhere equal to a, and $F_{x_{1}, u_{1}}=\Theta\left(x_{1}, u_{1}\right)$ be norm preserving 
on $E_{1}$ with $F_{x_{1}, u_{1}}(-v)=-F_{x_{1}, u_{1}}(v)$ for all $v \in E_{1}$. Then we have

$$
\left\|F_{x_{0}, u_{0}}-F_{x_{1}, u_{1}}\right\|_{\infty}=\max _{v \in E_{1}}\left\{\left\|F_{x_{0}, u_{0}}(v)-F_{x_{1}, u_{1}}(v)\right\|_{E}\right\} .
$$

Furthermore,

$$
\begin{aligned}
\left\|F_{x_{0}, u_{0}}(v)-F_{x_{1}, u_{1}}(v)\right\|_{E} & =\left\|\mathbf{a}-F_{x_{1}, u_{1}}(v)\right\|_{E}, \\
\left\|F_{x_{0}, u_{0}}(-v)-F_{x_{1}, u_{1}}(-v)\right\|_{E} & =\left\|\mathbf{a}+F_{x_{1}, u_{1}}(v)\right\|_{E},
\end{aligned}
$$

implying that

$$
4=\left\|\mathbf{a}-F_{x_{1}, u_{1}}(v)\right\|_{E}^{2}+\left\|\mathbf{a}+F_{x_{1}, u_{1}}(v)\right\|_{E}^{2} \leq 2 \max \left\{\left\|\mathbf{a} \pm F_{x_{1}, u_{1}}(v)\right\|_{E}^{2}\right\} .
$$

Consequently,

$$
\left\|F_{x_{0}, u_{0}}-F_{x_{1}, u_{1}}\right\|_{\infty} \geq \sqrt{2} .
$$

As mentioned before, this implies that the range of $\Theta$ contains only constant functions or only norm preserving maps. Now we show that the assumption that the range of $\Theta$ contains only norm preserving maps that send antipodal points to antipodal points leads to a contradiction.

In fact, if the range of $\Theta$ contains only such maps, then for a fixed constant function $f$ on $[0,1]$ equal to $\mathbf{a} \in E_{1}$, we have

$$
\langle(T f)(x), u\rangle+\left\langle(T f)^{\prime}(x), v\right\rangle=\left\langle a, F_{(x, u)}(v)\right\rangle
$$

and

$$
\langle(T f)(x), u\rangle-\left\langle(T f)^{\prime}(x), v\right\rangle=\left\langle a, F_{(x, u)}(-v)\right\rangle .
$$

Therefore $\langle(T f)(x), u\rangle=0$ for all $u$ and $x$, and so $T f$ is zero. This completes the proof.

REMARK 2.3. We mention that we can also prove, following a similar strategy, that for a fixed $x \in[0,1]$ and $\psi \in E_{1}^{*}$, the map $\tau_{(x, \psi)}: E_{1}^{*} \rightarrow E_{1}^{*}$ such that

$$
\tau_{(x, \psi)}(\varphi)=\psi_{1}
$$

is constant. This result is stated in Lemma 3.2 of the next section.

3. Surjective isometries of $C^{(1)}([0,1], E)$. In this section we establish that surjective isometries on $C^{(1)}([0,1], E)$ are composition operators. First, we prove preliminary results about surjective isometries on these spaces. The space $E$ is a finite-dimensional Hilbert space. The Riesz Representation Theorem allows us to associate a unique unit vector to each functional in $E_{1}^{*}$. Then we represent $\tau:[0,1] \times E_{1} \times E_{1} \rightarrow[0,1] \times E_{1} \times E_{1}$ given by $\tau(x, u, v)=\tau\left(x, u_{1}, v_{1}\right)$ with $u, v, u_{1}, v_{1}$ corresponding to $\varphi, \psi, \varphi_{1}, \psi_{1}$ respectively.

LEMma 3.1. If $E$ is a finite-dimensional real Hilbert space and $T$ is a surjective isometry on $C^{(1)}([0,1], E)$ then $T$ maps constant functions to constant functions. 
Proof. We assume that there exists a constant function $f \in C^{(1)}([0,1], E)$ with $f(t)=\mathbf{a}$, a vector in $E$, such that $T f$ is not constant. This means there exists $x_{0} \in[0,1]$ such that $(T f)^{\prime}\left(x_{0}\right) \neq 0_{E}$. We choose a vector $v_{0}$ in $E_{1}$ orthogonal to $(T f)^{\prime}\left(x_{0}\right)$, i.e. $\left\langle(T f)^{\prime}\left(x_{0}\right), v_{0}\right\rangle=0$. We set $\tau\left(x_{0}, u, v\right)=$ $\left(x_{1}, u_{1}, v_{1}\right)$. Then

$$
\left\langle(T f)\left(x_{0}\right), u\right\rangle+\left\langle(T f)^{\prime}\left(x_{0}\right), v\right\rangle=\left\langle\mathbf{a}, u_{1}\right\rangle .
$$

Lemma 2.2 implies that

$$
\left\langle(T f)\left(x_{0}\right), u\right\rangle+\left\langle(T f)^{\prime}\left(x_{0}\right), v_{0}\right\rangle=\left\langle\mathbf{a}, u_{1}\right\rangle .
$$

Therefore $\left\langle(T f)\left(x_{0}\right), u\right\rangle=\left\langle\mathbf{a}, u_{1}\right\rangle$ and $\left\langle(T f)^{\prime}\left(x_{0}\right), v\right\rangle=0$ for every $v$. This contradicts our initial assumption that $(T f)^{\prime}\left(x_{0}\right) \neq 0_{E}$, and completes the proof.

For a fixed $x \in[0,1]$ and $v \in E_{1}$, we define $\tau_{(x, v)}: E_{1} \rightarrow E_{1}$ by

$$
\tau_{(x, v)}(u)=v_{1} \text { provided that } \tau(x, u, v)=\left(x_{1}, u_{1}, v_{1}\right) .
$$

LEMMA 3.2. If $E$ is a finite-dimensional real Hilbert space then, for fixed $x \in[0,1]$ and $v \in E_{1}, \tau_{(x, v)}$ is constant.

Proof. We follow the steps in the proof of Lemma 2.2 with the following modification. We consider functions of the form $f(t)=\left(t-x_{1}\right) \mathbf{a}$ with a representing some unit vector in $E$, and set $F(u)=v_{1}$ with $u$ and $v_{1}$ associated with the functions $\varphi$ and $\psi_{1}$, respectively. A similar strategy to that followed in Lemma 2.2 allows us to conclude that either $F$ is constant or $(T f)^{\prime}$ is zero. If $(T f)^{\prime}$ is zero, then $T f$ is constant. Lemma 3.1 and the surjectivity of $T$ imply that $f$ must be constant. This contradiction completes the proof.

Lemma 3.3. If $E$ is a finite-dimensional real Hilbert space, $x$ and $x_{1}$ are such that $\tau(x, u, v)=\left(x_{1}, u_{1}, v_{1}\right)$, and $f \in C^{(1)}([0,1], E)$, then $f\left(x_{1}\right)=0_{E}$ implies that $(T f)(x)=0_{E}$.

Proof. Equation (2.1) reduces to

$$
\langle(T f)(x), u\rangle+\left\langle(T f)^{\prime}(x), v\right\rangle=\left\langle f^{\prime}\left(x_{1}\right), v_{1}\right\rangle .
$$

Now considering $u_{0} \in E_{1}$, Lemmas 2.1 and 3.2 imply that

$$
\left\langle(T f)(x), u_{0}\right\rangle+\left\langle(T f)^{\prime}(x), v\right\rangle=\left\langle f^{\prime}\left(x_{1}\right), v_{1}\right\rangle .
$$

Therefore $\left\langle(T f)(x), u-u_{0}\right\rangle=0$. Since $u_{0}$ is chosen arbitrarily in $E_{1}$ we conclude that $T(f)(x)=0_{E}$.

LEMMA 3.4. If $E$ is a finite-dimensional real Hilbert space and $T$ is a surjective isometry on $C^{(1)}([0,1], E)$, then there exists a surjective isometry $U$ on $E$ and a homeomorphism $\sigma$ on the interval $[0,1]$ such that

$$
T(f)(t)=U(f(\sigma(t)))
$$

for every $f \in C^{(1)}([0,1], E)$. 
Proof. We define $U(v)=T(\tilde{v})(0)$ with $\tilde{v}$ representing the constant function equal to $v$. Since $T$ is a surjective isometry, $U$ is also a surjective isometry on $E$. Given $f \in C^{(1)}([0,1], E)$ and $x_{1} \in[0,1]$ we denote by $f_{1}$ the function given by $f_{1}(t)=f(t)-f\left(x_{1}\right)$. Lemma 3.3 implies that $T\left(f_{1}\right)(x)=0_{E}$. Therefore

$$
T(f)(x)=U\left(f\left(x_{1}\right)\right) .
$$

We set $\sigma(x)=x_{1}$; Lemmas 1.4 and 2.1 imply that $\sigma$ is a homeomorphism.

TheOREM 3.5. If $E$ is a finite-dimensional real Hilbert space, then $T$ is a surjective isometry on $C^{(1)}([0,1], E)$ if and only if there exists a surjective isometry on $E$ such that for every $f$,

$$
T(f)(x)=U(f(\sigma(x)))
$$

with $\sigma=$ Id or $\sigma=1-$ Id.

Proof. It is clear that a composition operator of the form described in the theorem is a surjective isometry on $C^{(1)}([0,1], E)$. Conversely, if $T$ is a surjective isometry then Lemma 3.4 asserts the existence of a surjective isometry $U$ on $E$ and a homeomorphism $\sigma$ on the interval $[0,1]$ such that

$$
T(f)(t)=U(f(\sigma(t)))
$$

for every $f \in C^{(1)}([0,1], E)$. In particular, if $f(x)=x \mathbf{a}$ with a an arbitrary vector in $E$, then $T(f)(x)=\sigma(x) U(\mathbf{a})$. This implies that $\sigma$ is continuously differentiable. Similar considerations applied to $T^{-1}$ imply that $\sigma^{-1}$ is also continuously differentiable. Therefore $\sigma$ is a diffeomorphism of $[0,1]$. Since $\|T(f)\|_{1}=\max _{x}\left\{\|T f(x)\|_{E}+\left\|(T f)^{\prime}(x)\right\|_{E}\right\}$ and $T f(x)=U f(\sigma(x))$ with $U$ an isometry on $E$, we have

$$
\begin{aligned}
\|T f\|_{1} & =\max \left\{\|U f(\sigma(x))\|_{E}+\left\|U f^{\prime}(\sigma(x))\right\|_{E}\left|\sigma^{\prime}(x)\right|\right\} \\
& =\max \left\{\|f(\sigma(x))\|_{E}+\left\|f^{\prime}(\sigma(x))\right\|_{E}\left|\sigma^{\prime}(x)\right|\right\}
\end{aligned}
$$

and

$$
\|f\|_{1}=\max \left\{\|f(x)\|_{E}+\left\|f^{\prime}(x)\right\|_{E}\right\}=\left\|f\left(x_{0}\right)\right\|_{E}+\left\|f^{\prime}\left(x_{0}\right)\right\|_{E}
$$

for some $x_{0} \in[0,1]$. Therefore $\left|\sigma^{\prime}\left(\sigma^{-1}\left(x_{0}\right)\right)\right| \leq 1$. On the other hand, $T^{-1}(f)(x)=U^{-1} f\left(\sigma^{-1}(x)\right)$ and

$$
\begin{aligned}
r l\left\|T^{-1} f\right\|_{1} & =\max \left\{\left\|U^{-1} f\left(\sigma^{-1}(x)\right)\right\|_{E}+\left\|U^{-1} f^{\prime}\left(\sigma^{-1}(x)\right)\right\|_{E}\left|\left(\sigma^{-1}\right)^{\prime}(x)\right|\right\} \\
& =\max \left\{\left\|f\left(\sigma^{-1}(x)\right)\right\|_{E}+\left\|f^{\prime}\left(\sigma^{-1}(x)\right)\right\|_{E}\left|\left(\sigma^{-1}\right)^{\prime}(x)\right|\right\} .
\end{aligned}
$$

Therefore $\left|\left(\sigma^{-1}\right)^{\prime}\left(\sigma\left(x_{0}\right)\right)\right|=1 /\left|\sigma^{\prime}\left(x_{0}\right)\right| \leq 1$ and so $\left|\sigma^{\prime}\left(x_{0}\right)\right| \geq 1$. To conclude that $\left|\sigma^{\prime}(x)\right|=1$ for all $x$, we need to show that for every $x \in[0,1]$ there exists $f$ such that $\|f\|_{1}=\|f(x)\|_{E}+\left\|f^{\prime}(x)\right\|_{E}$ and $\left\|f^{\prime}(x)\right\|_{E} \neq 0$. We consider 
$f_{x}(t)=\lambda_{x}(t) \mathbf{a}$ with a a unit vector in $E$ and $\lambda_{x}$ given as in (1.1)

$$
\lambda_{x}(t)= \begin{cases}-\frac{1}{2}\left(x^{2}-t^{2}\right)+(x-1)(x-t) & \text { for } 0 \leq t \leq x, \\ -\frac{1}{2}\left(t^{2}-x^{2}\right)+(x+1)(t-x) & \text { for } x \leq t \leq 1 .\end{cases}
$$

Hence $\left|\sigma^{\prime}\right|=1$ and so $\sigma=\operatorname{Id}$ or $=1-\operatorname{Id}$.

REMARK 3.6. If the range space $E$ is an infinite-dimensional separable Hilbert space then there are nonsurjective isometries. Let $\left\{e_{n}\right\}_{n \in \mathbb{N}}$ be an orthonormal basis and $U$ be the operator defined by $U\left(e_{n}\right)=e_{2 n}$. The isometry $T: C^{(1)}([0,1], E) \rightarrow C^{(1)}([0,1], E)$ given by $T(f)(x)=U(f(x))$ is not surjective. It is not clear, whenever $E$ is finite-dimensional, if there are isometries on $C^{(1)}([0,1], E)$ which are not surjective.

Theorem 3.5 was stated for range spaces that are finite-dimensional Hilbert spaces over the reals, and we now extend our characterization to finite-dimensional Hilbert spaces over the complex numbers.

Corollary 3.7. If $E$ is a finite-dimensional complex Hilbert space, then $T$ is a surjective isometry on $C^{(1)}([0,1], E)$ if and only if there exists a surjective isometry $U$ on $E$ such that, for every $f$,

$$
T(f)(x)=U(f(\sigma(x)))
$$

with $\sigma=\operatorname{Id}$ or $\sigma(x)=1-\mathrm{Id}$.

Proof. The space $E$ is equipped with an inner product over $\mathbb{C}$, denoted by $\langle\cdot, \cdot\rangle$. This inner product induces a norm on $E$, denoted by $\|\cdot\|$, and the norm $\|\cdot\|_{1}$ is defined on the space $C^{(1)}([0,1], E)$. We define another inner product $(\cdot, \cdot)$ on $E$ by

$$
(u, v)=\operatorname{Re}\langle u, v\rangle .
$$

The space $E$ with multiplication only by real scalars and equipped with this real inner product $(\cdot, \cdot)$, is a Hilbert space, denoted by $\widetilde{E}$. The induced norm is denoted by $\|\cdot\|$ and

$$
\|f\|_{1}=\sup _{x \in[0,1]}\left\{\|f(x)\|+\left\|f^{\prime}(x)\right\|\right\}
$$

is the corresponding norm on $C^{(1)}([0,1], \widetilde{E})$. The identity map id $:(E,\|\cdot\|) \rightarrow$ $(E,\|\cdot\|)$ is real linear. Furthermore, given $u \in E$ we have

$$
\|u\|^{2}=(u, u)=\operatorname{Re}\langle u, u\rangle=\|u\|^{2} .
$$

Consequently, $(\widetilde{E},\|\cdot\|)$ and $(E,\|\cdot\|)$ are linearly isometric as real Banach spaces. If $T$ is a surjective isometry on $C^{(1)}([0,1], E)$, then $\widetilde{T}$, on $C^{(1)}([0,1], \widetilde{E})$, given by $\widetilde{T}(f)=T(f)$ is also a surjective isometry. In fact,

$$
\|\widetilde{T} f\|_{1}=\sup _{t \in[0,1]}\left\{\|\widetilde{T} f(t)\|+\left\|(\widetilde{T} f)^{\prime}(t)\right\|\right\}=\sup _{t \in[0,1]}\left\{\|f(t)\|+\left\|f^{\prime}(t)\right\|\right\}=\|f\|_{1} .
$$


Theorem 3.5 now asserts that there exists a real isometry $U$ on $\widetilde{E}$ and $\sigma=\mathrm{Id}$ or $1-\mathrm{Id}$ so that $\widetilde{T}(f)(t)=U(f(\sigma(t)))$. Then it follows that $T(f)(t)=$ $U(f(\sigma(t)))$. It also follows that $U$ is a complex linear isometry by considering constant functions. This concludes the proof.

4. Generalized bi-circular projections on $C^{(1)}([0,1], E)$. In this section we give a characterization of all generalized bi-circular projections on $C^{(1)}([0,1], E)$ with $E$ a finite-dimensional complex Hilbert space. We starting by reviewing the definition of generalized bi-circular projection.

Definition 4.1 (cf. [8]). A bounded linear projection $P$ on $C^{(1)}([0,1], E)$ is said to be a generalized bi-circular projection if and only if there exists a modulus 1 complex number $\lambda$, different from 1 , so that $P+\lambda(\operatorname{Id}-P)$ is an isometry $T$ on $C^{(1)}([0,1], E)$.

The isometry $T$ must satisfy the following operator quadratic equation:

$$
T^{2}-(1+\lambda) T+\lambda \operatorname{Id}=0 .
$$

Since $T$ is a surjective isometry, Theorem 3.5 implies the existence of a surjective isometry $U$ on $E$ such that

$$
U^{2} f(x)-(1+\lambda) U(f(\sigma(x))+\lambda f(x)=0 .
$$

Therefore if $\lambda=-1$ then $U^{2}=\operatorname{Id}$ and $P$ is the average of the identity with an isometric reflection $R(f)(x)=U(f(\sigma(x)))$. If $\lambda \neq-1$, then $\sigma(x)=x$ for every $x \in[0,1]$ and $U^{2}-(1+\lambda) U+\lambda \operatorname{Id}=0$. Hence

$$
P(f)=\frac{U-\lambda \mathrm{Id}}{1-\lambda} f(x) \text {. }
$$

We summarize the previous considerations in the following proposition.

Proposition 4.2. Let $E$ be a finite-dimensional complex Hilbert space. Then $P$ is a generalized bi-circular projection on $C^{(1)}([0,1], E)$ if and only if there exists a generalized bi-circular projection $P_{E}$ on $E$ so that $\operatorname{Pf}(x)=$ $P_{E}(f(x))$.

REMARK 4.3. We wish to thank the referee for several helpful suggestions that resulted in a substantial improvement of this paper. The referee also suggested that the proof of our main result could be shortened by using results by Jarosz and Pathak in [9].

\section{References}

[1] R. Arens and J. Kelley, Characterization of spaces of continuous functions over compact Hausdorff spaces, Trans. Amer. Math. Soc. 62 (1947), 499-508.

[2] S. Banach, Théorie des opérations linéaires, Warszawa, 1932. 
[3] F. Botelho and J. E. Jamison, Generalized bi-circular projections on $C(\Omega, X)$, Rocky Mountain J. Math. (2008), to appear.

[4] M. Cambern, Isometries of certain Banach algebras, Studia Math. 25 (1965), 217225.

[5] M. Cambern and V. D. Pathak, Isometries of spaces of differentiable functions, Math. Japon. 26 (1981), 253-260.

[6] N. Dunford and J. T. Schwartz, Linear Operators Part I: General Theory, Interscience, New York, 1958.

[7] R. Fleming and J. Jamison, Isometries on Banach Spaces: Vol. I, Function Spaces, Vol. II, Vector-Valued, Function Spaces, Chapman \& Hall, 2003, 2007.

[8] M. Fosner, D. Ilišević and C. K. Li, G-invariant norms and bicircular projections, Linear Algebra Appl. 420 (2007), 596-608.

[9] K. Jarosz and V. Pathak, Isometries between function spaces, Trans. Amer. Math. Soc. 305 (1988), 193-206.

[10] K. S. Lau, A representation theorem for isometries of $C(X, E)$, Pacific J. Math. 60 (1975), 229-223.

[11] K. de Leeuw, Banach spaces of Lipschitz functions, Studia Math. 21 (1961), 55-66.

[12] N. V. Rao and A. K. Roy, Linear isometries on some function spaces, Pacific J. Math. 38 (1971), 177-192.

[13] E. Spanier, Algebraic Topology, McGraw-Hill, 1966.

[14] L. L. Stacho and B. Zalar, Bicircular projections and characterization of Hilbert spaces, Proc. Amer. Math. Soc. 132 (2004), 3019-3025.

[15] M. Stone, A general theory of spectra, II, Proc. Nat. Acad. Sci. U.S.A. 27 (1941), 83-87.

Department of Mathematical Sciences

The University of Memphis

Memphis, TN 38152, U.S.A.

E-mail: mbotelho@memphis.edu jjamison@memphis.edu

Received June 2, 2008

Revised version October 14, 2008 